\title{
WIDE-FIELD WASHINGTON PHOTOMETRY OF THE NGC 5128 GLOBULAR CLUSTER SYSTEM. I. THE DATABASE
}

\author{
Gretchen L. H. Harris ${ }^{1,2}$ \\ Department of Physics, University of Waterloo, Waterloo ON N2L 3G1, Canada; glharris@astro.uwaterloo.ca \\ Doug GeISLER ${ }^{1}$ \\ Universidad de Concepción, Departamento de Fisica, Casilla 160-C, Concepción, Chile; doug@kukita.cfm.udec.cl \\ William E. Harris ${ }^{2}$ \\ Department of Physics and Astronomy, McMaster University, Hamilton ON L8S 4M1, Canada; harris@physics.mcmaster.ca \\ BRIAN P. SCHMIDT \\ Research School of Astronomy and Astrophysics, Australian National University, Weston ACT 2611, Australia; \\ brian@mso.anu.edu.au \\ JAMES E. Hesser \\ Herzberg Institute of Astrophysics, National Research Council of Canada, 5071 West Saanich Road, \\ Victoria, BC V9E 2E7, Canada; jim.hesser@nrc.ca \\ AND \\ Mike Reid, ${ }^{3}$ Margaret Milne, ${ }^{4}$ Scott C. Hulme, and Tyler T. Kidd \\ Department of Physics, University of Waterloo, Waterloo ON N2L 3G1, Canada \\ Received 2003 October 14; accepted 2004 April 12
}

\begin{abstract}
To investigate the global properties of the globular cluster system (GCS) around NGC 5128, the central giant elliptical galaxy in the nearby Centaurus group, we have obtained deep CCD imaging for an area of almost $2 \mathrm{deg}^{2}$ centered on the galaxy. Our data, in the $C M T_{1}$ Washington photometric system, reach an approximate limiting magnitude of $T_{1} \simeq R \simeq 22$ and contain magnitudes, colors, and coordinates for more than 100,000 objects. Of these, the vast majority (about 99\%) are either foreground stars or faint background galaxies; the old-halo globular clusters make up the remaining tiny fraction of the sample. Our database, however, provides the material for understanding the large-scale features of the GCS, including its metallicity distribution (MDF), luminosity distribution, and spatial structure.
\end{abstract}

Key words: galaxies: individual (NGC 5128) — galaxies: stellar content — globular clusters: general

On-line material: machine-readable table

\section{INTRODUCTION}

NGC 5128 is the dominant giant elliptical galaxy in the Centaurus group of galaxies just $4 \mathrm{Mpc}$ from the Milky Way. It is easily the closest giant $\mathrm{E}$ galaxy amenable to detailed study, ${ }^{5}$ and as such it affords a unique opportunity to investigate at close range the characteristics of its oldest stellar populations (the old red giant halo stars and globular clusters). Thanks to a recent series of Hubble Space Telescope photometric studies, much has now been learned about the metallicity distribution of the old-halo stars (Soria et al. 1996; Harris et al. 1999; Harris \& Harris 2000; Harris \& Harris 2002; Marleau et al. 2000; Rejkuba et al. 2004); but, by contrast, the

${ }^{1}$ Visiting Observer, Cerro Tololo Inter-American Observatory, operated by the Association of Universities for Research in Astronomy, Inc., under cooperative agreement with the National Science Foundation.

${ }^{2}$ Visiting Fellow, Research School of Astronomy and Astrophysics, Australian National University, Weston ACT 2611, Australia.

${ }^{3}$ Current address: Department of Physics and Astronomy, McMaster University, Hamilton ON L8S 4M1, Canada.

${ }^{4}$ Current address: Department of Physics and Astronomy, University of Victoria, Victoria BC V8W 3P6, Canada.

${ }^{5}$ Maffei 1, an E galaxy of somewhat lower luminosity than NGC 5128 and at a similar distance, is deeply embedded in the heavy obscuration of the Galactic plane $\left(A_{V}=5.1 \mathrm{mag}\right.$; see Davidge \& van den Bergh 2001) and is considerably more difficult to study. large-scale characteristics of its globular cluster system (GCS) are not as well studied.

The first globular cluster in NGC 5128 was found serendipitously by Graham \& Phillips (1980) from its nonstellar appearance on photographic images. Painstaking examination of photographic plates, combined with radial velocity measurements, led to discoveries of more clusters in the next few years. Wide-field star counts and photographic photometry were also carried out, and by the end of the 1980s a solid beginning had been made outlining the features of the system and identifying many individual bright clusters (van den Bergh et al. 1981; Harris et al. 1984a; Harris et al. 1984b; Hesser et al. 1984, 1986; Sharples 1988; Harris et al. 1988). A first study of the important metallicity distribution function (MDF) was made through Washington system $C M T_{1} T_{2}$ photometry of 62 individual globular clusters by Harris et al. (1992, hereafter H92). This discussion gave the first clear indication that the MDF was very broad, comparable to other giant E systems, and with roughly equal numbers of metal-poor and metal-rich clusters, if reinterpreted according to the "bimodality" paradigm that was developed in subsequent years (e.g., Zepf \& Ashman 1993; Peng et al. 2004, among many others).

More recently, Rejkuba (2001) has obtained UV photometry of 78 additional cluster candidates, and Peng (2003) and Peng et al. (2004) have obtained radial velocities and UBVRI 
imaging for 138 clusters, of which 102 are newly identified. From HST imaging with the WFPC2 and STIS cameras, structural measurements and King-model profiles have been published for a total of 43 individual globular clusters (Holland et al. 1999; Harris et al. 2002), and it has even been possible to produce a color-magnitude diagram for one of them (Harris et al. 1998). There is no other giant $E$ galaxy for which information on globular clusters at this level of detail is currently possible.

Despite significant gains in these various directions, however, we still lack a quantitative broad-scale understanding of many features of the GCS, including the total cluster population, spatial distribution in the halo, or an MDF based on a large statistical sample. Symptomatic of our lack of understanding of the NGC 5128 GCS is that there is no comprehensive CCD-based study of its luminosity function (GCLF) which can be compared with more distant systems. In principle, the new generations of wide-field CCD array cameras provide the basic tools we need for such work. But for several reasons, as follows, NGC 5128 is a surprisingly difficult target for this kind of study:

1. NGC 5128 is at moderately low Galactic latitude $(b=$ $\left.19^{\circ} .4\right)$ and at a longitude $\left(l=309^{\circ} .5\right)$ only $\sim 50^{\circ}$ from the Galactic center. Thus, although its foreground reddening of $E(B-V)=$ 0.11 is not large, there is considerable contamination by both foreground field stars and faint, small background galaxies. These contaminants vastly outnumber the NGC 5128 globular clusters, and, to compound the problem, a high fraction of them have magnitudes and colors quite similar to the clusters.

2. The effects of field contamination are made worse by the fact that NGC 5128 is so nearby that its halo is very spread out in angular size, making its GCS population quite "dilute" across the sky. Yet it is distant enough that (at least with groundbased imaging) the clusters cannot be easily distinguished from field stars or background galaxies by virtue of morphological shape and size.

3. Finally, the GCS in NGC 5128 is not a "high specific frequency" system, making it even harder to see the total cluster population against the field. The wide-field photographic star counts performed two decades ago by Harris et al. (1984a) indicated that it has perhaps $\sim 1000$ globular clusters in total, corresponding to a specific frequency $S_{N} \sim 2$, much smaller than in the more cluster-rich Virgo ellipticals (Harris 2001). But the estimated total population is itself very poorly known.

In short, NGC 5128 presents us with the tantalizing potential to understand in detail the GCS of a large elliptical, but the necessary measurements are coupled with very nearly the worst possible set of observational circumstances (see Harris 1983; Hesser et al. 1984).

Further observational progress on this intriguing and challenging system now requires more comprehensive photometric studies covering a wide field, in metallicity-sensitive color indices and with deep enough limiting magnitude to reach the majority of its clusters. In this paper we describe new observations of this type obtained with the Big Throughput Camera (BTC) at the Cerro Tololo Inter-American Observatory $4 \mathrm{~m}$ telescope. In a subsequent discussion (Harris et al. 2003, hereafter Paper II) we will use the data to discuss the largescale properties of the cluster system: its spatial structure, metallicity distribution, and luminosity function.

\section{OBSERVATIONAL MATERIAL}

In the following sections we describe new wide-field imaging data taken in the Washington $C M T_{1}$ photometric system.

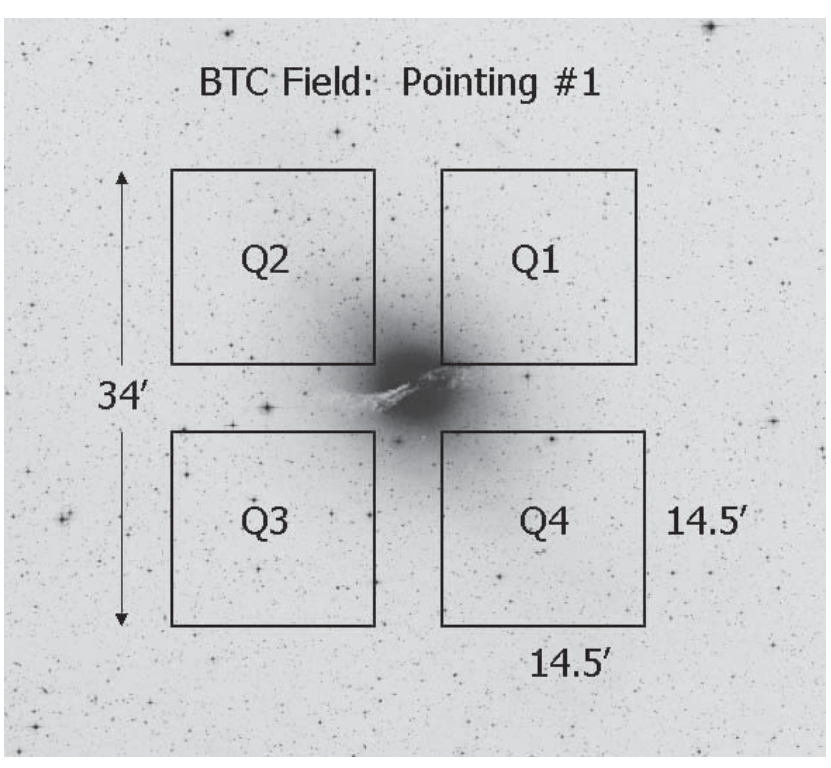

FIG. 1.-Outline of the BTC field of view and dimensions. The location of our first target field (pointing 1) is shown. North is at the top, and east at the left.

We chose this system because it can be used to construct photometric indices (particularly $C-T_{1}$ ) that are far more metallicity-sensitive for old stellar populations than other frequently used colors, such as $V-I$, and because the data would be closely compatible with our previous study (H92) and with Washington photometry of other GCSs. In the following text we will use the symbols $(C, M, R)$ to denote the three filters used with the BTC, while we use $(c, m, r)$ to denote the instrumental magnitudes (not yet transformed to the standard Washington system) derived from the photometry.

The BTC (now decommissioned) comprised an open mosaic of four thinned Tek CCDs, each $2048 \times 2048$ (Wittman et al. 1998). The mean scale of the camera is 0.424 pixel $^{-1}$ determined from our astrometric WCS solutions (see below). Thus, each of the four CCDs covered $14.5 \times 14.5$ on the sky. The mosaic is not "filled," with 760 pixel (5.4) gutters between each adjacent pair of CCDs, making the total span of the field equal to 34.3 . The scale and orientation of the array are shown in Figure 1: quadrants 1, 2, 3, and 4 are in the northwest, northeast, southeast, and southwest corners, respectively. The camera was operated at a gain setting of $2.44 e^{-} / d n$ and with a saturation limit of 60,000 dn. Filters used were the Washington $C$ and $M$ and a broadband Kron-Cousins $R$; Geisler (1986) has shown that the $R$ filter accurately reproduces the narrower Washington $T_{1}$ index and achieves a gain in efficiency of a factor of 3 . The resulting calibrated colors and magnitudes will therefore be on the Washington $C M T_{1}$ system.

We obtained sets of images for nine different array positions around NGC 5128 during two nights in 1998 (May 11-13) and two nights in 1999 (May 26-28). We will refer to these field locations as "pointings" 1 through 9; they were located as shown in Figures 2 and 3. Pointing 1 was the innermost and was positioned with the galaxy nucleus in the central gap of the mosaic. Four "outer" pointings $(6,7,8$, and 9$)$ were butted adjacent to pointing 1 . Four more "middle-ring" pointings (2, 3,4 , and 5) were positioned such that each would mutually overlap with at least one other pointing on at least two of its quadrants, while also covering as much of the interchip gaps as possible. In this way, the internal photometry from all the CCDs could eventually be tied closely together through the 


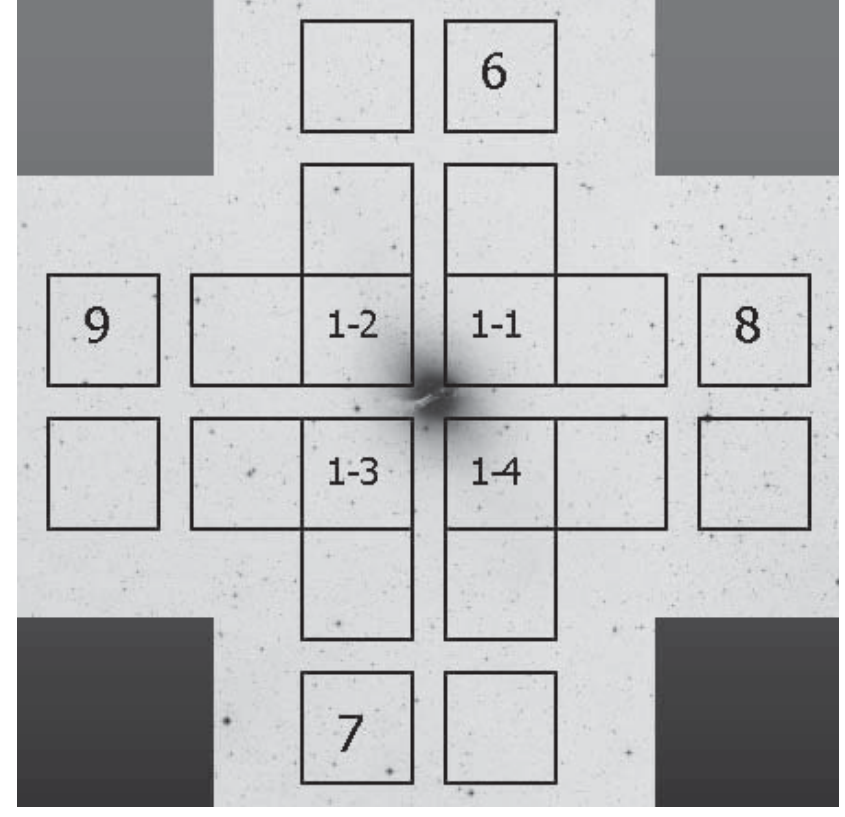

FIG. 2.- Locations of the central pointing 1, and the four outer fields 6,7 , 8 , and 9 . The field shown is $1^{\circ} .9$ across

overlap regions. When all the fields were combined, they gave nearly complete areal coverage for a box-shaped region of sky 1.2 across centered on NGC 5128, and partial coverage extending to 1.7 across. In all, the total area covered by all our pointings was $1.9 \mathrm{deg}^{2}$.

For each pointing we obtained five raw images: three long exposures in $C(2000 \mathrm{~s}), M(500 \mathrm{~s})$, and $R(500 \mathrm{~s})$, and two short exposures of $100 \mathrm{~s}$ each in $M$ and $R$. The BTC was not a highly blue-sensitive detector, and globular clusters are moderately red objects, so in practice the effective limiting magnitude in the long-exposure $C$ image was not quite as deep as in the short $M$ and $R$. Consequently, as is usually the case, our multicolor data for the detected objects are ultimately limited by the $C$ images. By contrast, the limits for the spatial distribution and luminosity function analyses are set by the long-exposure $M$ and $R$ images, which reach 1-2 mag deeper than $C$. With five exposures per pointing, nine pointings, and four CCD images per mosaic, we had a total raw database of 180 images to correlate.

Unfortunately, none of the four nights of observation was photometric, and so Washington system standard stars were not observed (we will return to the issue of absolute calibration below). Poor weather conditions during the 1998 observing run meant that we could obtain only four suitable exposures during the first night (short and long $R$ for pointings 1 and 2 ) and none on the second night. And the weather conditions in 1999, though better, were not good enough to allow time to repeat those pointings. Thus, 41 of the 45 exposures were obtained during the 1999 May run. The seeing quality varied from 0.9 at best up to 2".3 at worst; but for most exposures the seeing was between 1 ".2 and 1 ."5.

The range of seeing quality can be seen in Figure 4 in which the FWHM is plotted for each chip and each filter of the full data set. For the most part, FWHM values are between 2 and 4 pixels $\left(\sim 0.9-1{ }^{\prime \prime} .7\right)$, and the data for a given pointing usually span $\sim 75 \%$ of this full range. In pointings 1 and 2 , where the $R$ exposures were taken in 1998, the FWHM values are on average twice those for the corresponding $M$ and $C$ images.

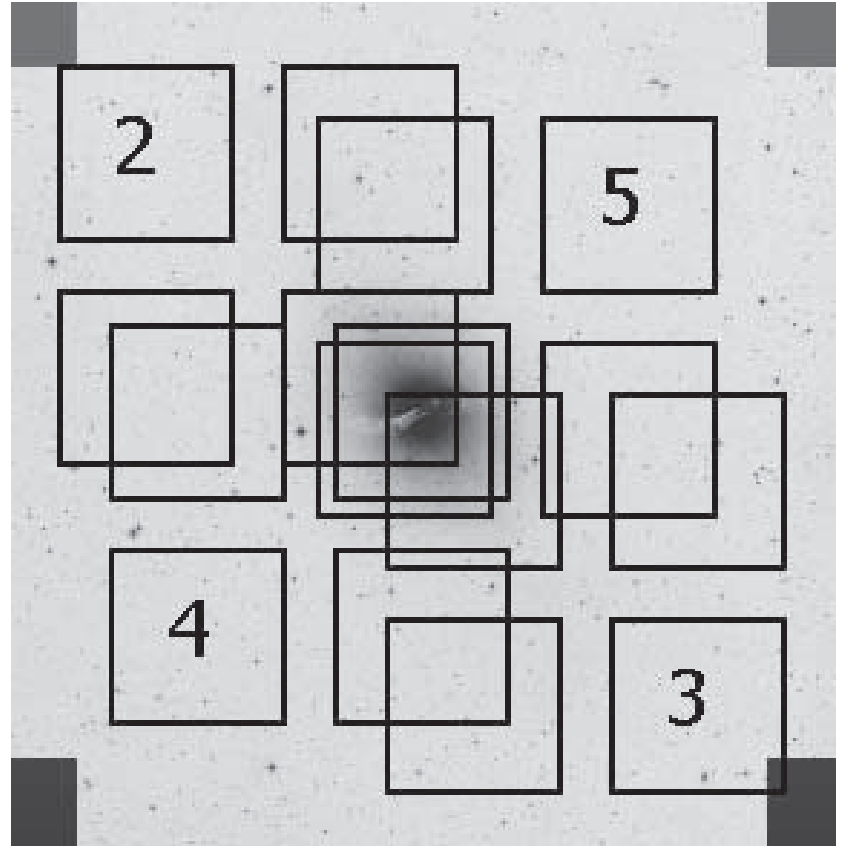

FIG. 3.-Locations of the "middle-ring" pointings 2, 3, 4, and 5. The field shown is 1.3 across.

For pointings $3-8$, the range of FWHM values is considerably smaller, with the puzzling exception of the long and short exposures on chip 1; this could be due to a slight alignment or focus problem, but no notes of special problems were made at the time of observations.

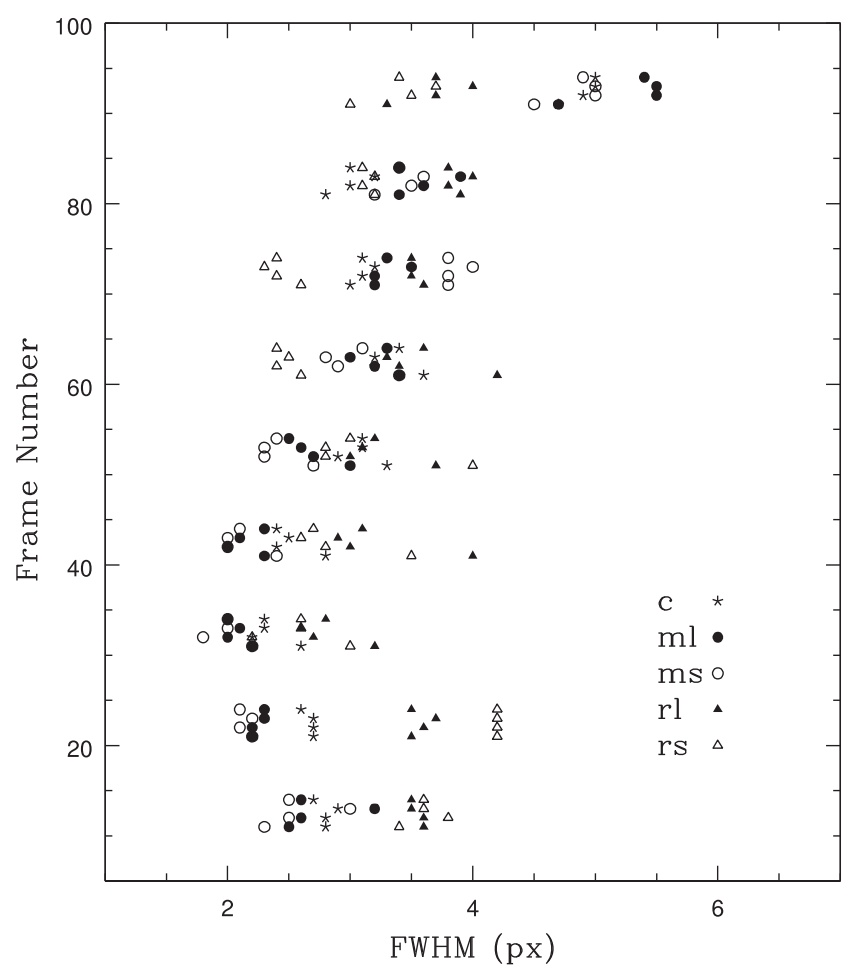

FIG. 4.-Approximate FWHM values in pixels for all four chips on all nine exposures. The "frame number" plotted on the $y$-axis is the label for the pointing and chip; for example, pointing 1 and chip 4 will have frame number 14. $C$ frames are plotted as asterisks, $M$ long and short are filled and open circles, respectively, while $R$ long and short are plotted as filled and open triangles. 
Finally, the FWHMs for the $C$ and $M$ exposures for pointing 9 are the largest of all and reflect a deteriorating seeing quality at the end of the night.

Many of the raw images $(\sim 60 \%)$ were also taken through small amounts of cloud cover. These differences in seeing and transparency mean that the limiting magnitudes differed quite noticeably from one frame to the next, an issue that will come into play during the later analysis.

\section{DATA REDUCTION AND PHOTOMETRY}

\subsection{Preparing the Photometry}

All frames were bias-corrected and flat-fielded through IRAF CCDRED. As described in $\S 3.2$, we first created "rectified" images corrected for image scale variations across each chip. PSF-fitting photometry was then carried out with DAOPHOT routines (Stetson 1992) through the normal sequence of FIND/PHOT/PSF/ALLSTAR. The point-spread function (PSF) was constructed separately for each chip and each exposure from typically 20 to 30 stars spread across the field.

In the few cases where the galaxy nucleus was on the chip and the bulge covered a substantial fraction of the chip area, the image was median filtered and the smoothed frame subtracted from the original, removing most of the strong background light gradient across the frame and improving the DAOPHOT/FIND object detection step.

Sky background values were lowest in the short (100 s) $M$ exposures and highest in the long $(500 \mathrm{~s}) R$, with the latter typically 10 times greater; representative values for good sky conditions are shown in Table 1 . In spite of the higher sky background for the long $R$ exposures, we note again that the limiting magnitude for this data set was set by the $C$ frames, as is typical in the Washington system (e.g., Geisler 1996).

Typically, each chip on each exposure contained 2000 to 5000 detected objects, a small enough number that the photometry was little affected by crowding. As will be shown below, the typical separation between each object and its nearest detected neighbor, regardless of magnitude, was about 10 pixels or larger, well beyond the FWHM of the PSF on any of the frames.

The multiply overlapping field locations and the different magnitude limits on each pointing make the effective limiting magnitude of the entire data set an extremely complex and rather choppy function of location across the field. For this reason, no artificial-star tests to determine completeness were done. However, the detection limit everywhere across the region is considerably deeper than the magnitude $T_{1} \simeq 20.5$ at which the GCLF turnover is expected. That is, our data should comfortably include the great majority of the old-halo globular clusters in NGC 5128, as intended.

All of our final BTC photometry consists of DAOPHOT/ ALLSTAR PSF-fitted magnitudes, calibrated through aperture photometry of brighter stars across the field and transformed into $C M T_{1}$ (the calibration procedure is described in $\S 4$ below). No objects were deleted from our photometry lists because of image shape or morphology. Many of the globular clusters in NGC 5128 appear starlike, or nearly so, under the seeing of $\sim 1 " .2-1$ ".5 that typifies our data. However, many others are physically extended enough to appear clearly nonstellar, so we cannot reject either stellar or nonstellar objects a priori. For example, the half-light radii for the sample of very luminous clusters studied by Harris et al. (2002) are typically 0 " 4 or less, corresponding to diameters of $\sim 8 \mathrm{pc}$. But some clusters, particularly the brighter outer-halo ones that have
TABLE 1

Typical Sky Background Values for CCD Exposures

\begin{tabular}{ccc}
\hline Filter & $\begin{array}{r}\text { Exposure Time } \\
(\mathrm{s})\end{array}$ & $\begin{array}{c}\text { Mean Sky } \\
\text { (counts) }\end{array}$ \\
\hline & 2000 & 2500 \\
& 100 & 1000 \\
& 500 & 4500 \\
$R$ long & 100 & 2000 \\
$R$ long & 500 & 9000 \\
\hline
\end{tabular}

${ }^{a}$ These are representative values, which indicate range and pattern of sky intensity but not exact values for most exposures.

been found individually by image morphology (e.g., van den Bergh et al. 1981; Hesser et al. 1984; Harris et al. 1984b; Rejkuba 2001), have more extended structures, and for these the PSF-fitted magnitudes will underestimate their total light. The actual amount of shortfall will depend on the cluster size but (as will be seen below) can reach $0.2 \mathrm{mag}$. For the bigger clusters, aperture photometry (as was used in H92) is the systematically better procedure, but it is subject to errors in cases where the object is crowded.

In short, there is no single ideal procedure for photometry of all clusters in this galaxy; they overlap in morphology and color with both the foreground stars and the small, faint background galaxies. Higher resolution ground-based imaging (say at $\sim 0$ ".5 resolution) would separate out many more cluster candidates, but the only truly definitive approaches to weeding them out are the much more time-consuming ones of radial velocity measurement (Hesser et al. 1986; Peng 2003) or the even more expensive process of resolving the clusters into stars with, e.g., HST (Harris et al. 1998). Our BTC survey is therefore intended to be useful primarily as a statistical database and as a starting point for future work on the individual clusters.

\subsection{Calibration of Coordinates}

To combine the data from all pointings readily and also to have positions adequate for future spectroscopic and imaging studies, we derived transformations of the $x y$ pixel coordinate system of each chip on each pointing to the world coordinate system (WCS). The USNO UCAC1 catalog (Zacharias et al. $2000)$, with its extensive reference grid of stars, was used to establish the WCS transformations. The matches were automated through a program that searched for similar triangles (Groth 1986) and then fitted linear transformations to the matched set of stars. These stars were then used to increase the order of the fitted transformation first to quadratic and finally to cubic, rematching the stars each time (Schmidt et al. 1998). Then the match list was put into the IRAF task CCMAP, for writing the WCS solution into the header using the nonstandard TNX projection. On average, 400 to 500 stars per CCD frame (or more than two stars per square arcminute) were used to define the coordinate transformations. The only particular problem encountered in this procedure was on the few frames containing the large central bulge of NGC 5128, where only 100 to 200 absolute reference stars were usable and somewhat bigger interpolation steps needed to be taken. However, it should be noted that these same inner frames overlapped heavily with many of the outer frames (see below for the description of the splicing procedure), and very little of the total area surveyed relied only on the coordinates from the innermost fields. 
The astrometric transformations showed that the precise image scale on each CCD is not constant across the $14^{\prime}$ chip width, changing by up to $8 \%$ from corner to corner particularly in the radial direction relative to the optical axis of the telescope. These scale nonuniformities, if ignored, can introduce internal scatter of a few percent in our instrumental photometry since the true area of each pixel projected on the sky is a function of location on the CCD. Before doing the final DAOPHOT photometry described above, we therefore used the WCS transformations to create rectified images, in all five exposures of each pointing, that had strictly uniform image scales and internal photometric zero points (i.e., all pixels in the rectified image have the same true area on the sky and area-corrected sensitivity). The internal quality of the UCAC1 catalog grid leads us to expect that our coordinate measurements will be good to $\simeq 0$ " 1 across the entire region. Our internal tests from the mutually overlapping pointings (see below) verify this expectation.

After the photometry was completed, the $x y$ coordinates for all objects on each filter, chip, and pointing were converted to a common local $x y$ system (that of the short- $R$ exposure) for that pointing. We used the IRAF tasks GEOMAP and GEOXYTRAN for this purpose. ${ }^{6}$ For each measured object on the short- $R$ frame we found the object closest to it in position on the other four frames, within a specified tolerance of a 2 pixel radius $\left(\sim 1^{\prime \prime}\right)$. We kept only objects that appeared in both $R$ and $M$, discarding any objects that appeared in only one color. The long and short exposure measurements in $M$ and $R$ were combined with a weighted average $m(a v)=$ $\left(m_{s} w_{s}+m_{l} w_{l}\right) /\left(w_{s}+w_{l}\right)$, where the weights $w$ are the inverse squares of the internal measurement uncertainties, $w_{i}=e_{i}^{-2}$. The result of this step was a complete and homogeneous set of instrumental magnitudes $(c, m, r)$, measurement uncertainties, and coordinates for all stars on each individual pointing.

\section{ASSEMBLING THE COMBINED DATA SET}

The next stage was to splice together all the mutually overlapping pointings onto a single homogeneous instrumental magnitude system. Between any two pairs of overlapping frames, we found anywhere from 115 to 950 moderately bright stars in common (with an average of 400 ) to define the frameto-frame internal magnitude scale differences $\Delta r, \Delta m, \Delta c$ and mean differences in the WCS-transformed coordinates $(\Delta \alpha, \Delta \delta)$. The various chips and pointings were merged this way into successively bigger "pieces" until all had been brought together into a single list.

The mean rms frame-to-frame scatter in $\Delta r, \Delta m, \Delta c$ for any one splicing step averaged $\sigma_{r}=0.027, \sigma_{m}=0.021$, and $\sigma_{c}=0.021 \mathrm{mag}$. These figures give useful average estimates of the internal precision of the PSF-fitting photometry for the brighter stars, and are consistent with the quoted errors from DAOPHOT/ALLSTAR (Fig. 9 below). The frame-to-frame coordinate shifts needed to place one frame onto the $(\alpha, \delta)$ system of an adjacent one averaged $|\Delta \alpha|=0.072,|\Delta \delta|=$ 0.100 and were never larger than 0.3 . These averages are consistent with the expected precision of our WCS solutions

\footnotetext{
${ }^{6}$ We note that all objects found on either the long- $R$ or short- $R$ frames were included in our discovery lists; however, because the long- $R$ images had many overexposed stars that were necessary for absolute coordinate calculation, we adopted the short- $R$ frames to define the local coordinate system and transformed all other exposures to them.
}

TABLE 2

CCD Zero-Point Differences in BTC Array

\begin{tabular}{ccccc}
\hline \hline Filter & $\begin{array}{c}\text { Chip 1 } \\
\text { (mag) }\end{array}$ & $\begin{array}{c}\text { Chip 2 } \\
\text { (mag) }\end{array}$ & $\begin{array}{c}\text { Chip 3 } \\
\text { (mag) }\end{array}$ & $\begin{array}{c}\text { Chip 4 } \\
\text { (mag) }\end{array}$ \\
\hline & 0.011 & 0.357 & 0.080 & $(0.000)$ \\
& 0.020 & 0.215 & 0.053 & $(0.000)$ \\
& 0.030 & 0.103 & 0.029 & $(0.000)$ \\
\hline
\end{tabular}

mentioned above. The rms star-to-star scatters averaged $\sigma_{\alpha}=0$."053, $\sigma_{\delta}=0$ ".062. In the later discussion we will describe other external tests of the global coordinate system accuracy.

Some of the individual CCD fields did not overlap with any of the others. Specifically, these were the quadrants 2-2 and 3-4 at the northeast and southwest corners of the main central region, and the eight outermost quadrants (6-1, 6-2, 7-3, 7-4, 8-1, 8-4, 9-2, and 9-3). Furthermore, these individual fields (all at large distances from the center of NGC 5128) did not contain any secondary photometric standards of their own (see below), and a special procedure was needed to bring them onto the photometric scale of the rest of the merged data set.

The approach we adopted for these outliers was to make sure that their instrumental magnitudes $(c, m, r)$ were on the same internal scale as the other three quadrants from the same pointing, since at least two of the other three quadrants in every pointing overlapped with the main (central) body of data. To do this, we needed to know the absolute zero-point differences (that is, intrinsic sensitivity differences) among the four CCD chips in the BTC mosaic. These in turn could be determined from within the inner region with its many multiply overlapping fields (for example, field 1-1 overlaps with all four CCDs in pointing 5, and field 1-3 overlaps with all four chips in pointing 4). We used these multiply overlapping quadrants to determine the mean chip-to-chip differences in the raw aperture photometry zero points, with results as listed in Table 2. It can be seen that chips 1,3 , and 4 all had much the same sensitivity (differing by at most $0.08 \mathrm{mag}$ ), but chip 2 was noticeably less sensitive, becoming worse toward bluer wavelength. By assumption, chip 4 (the most sensitive one) was set to $\Delta=0.000$, and we normalized the other three to it.

We believe that this procedure has been adequate to put these outlying fields on the same homogeneous photometric scales to within \pm 0.05 mag. This level of uncertainty is definitely worse than for the more closely knit inner fields, but, as will be seen in Paper II, this outlying material is used only for statistical purposes to help define the background levels.

One final "cleaning" step was applied to the combined photometry list before we moved on to the absolute calibration stage: four of the quadrants $(2-4,3-2,4-1$, and 5-3) contained the central bulge of NGC 5128. Within these, there were huge numbers of false or multiple detections generated by the very bright bulge light and by the dust lane crossing the nucleus. We have simply removed any objects in this nuclear region from our lists. Experimentation with various "exclusion boxes" centered on the nucleus led us to adopt an ellipse of semimajor axis 4.2 , axial ratio $b / a=0.50$, and major axis orientation angle $25^{\circ}$ north of west (putting its major axis parallel to the dust lane). Any objects within that ellipse, whether real or false, were deleted from the database. 


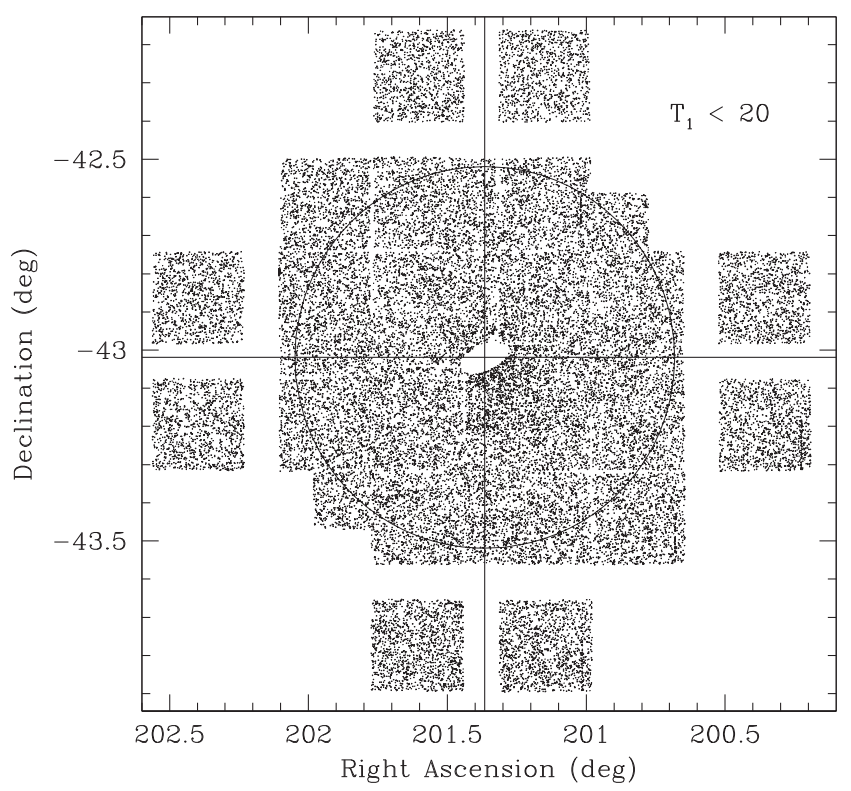

FIG. 5.-Locations of the $\sim 33,000$ detected objects brighter than $T_{1}=20.0$ in the entire BTC database. The center of NGC 5128 is marked with the horizontal and vertical lines, and the circle shown has a radius of 0.5 .

The final database contains 107,040 measured objects. In Figure 5 we show the coordinate distribution $(\alpha, \delta)$ for the $\sim 33,000$ brightest objects $\left(T_{1}<20\right)$ in the complete database. The small innermost exclusion ellipse is clearly visible, as are the eight outlying CCDs separate from the main body of data. The other two separate chips mentioned above (2-2 and 3-4) narrowly missed any overlaps and fitted almost precisely into the upper left and lower right corners. In total, our surveyed field spans a diameter of 1.7 , with the central "box" covering $1^{\circ} .2$ edge to edge. As shown in the figure, our data give us complete coverage of the NGC 5128 halo to more than 0.5 radius, equivalent to a projected radius of $35 \mathrm{kpc}$.

Although the total number of measured objects in our database is large, the actual degree of crowding is not. In Figure 6 the distribution of "nearest-neighbor" distances is shown for all the objects in the database. The most frequent separation is in the range $3^{\prime \prime}-6^{\prime \prime}$ (7-15 pixels), and almost no objects have neighbors within an arcsecond. (Because almost all the objects in the photometry list are "field" contaminants, their numbers to a given limiting magnitude are very much the same across the entire region, so the shape and peak of this histogram do not differ much from place to place.)

\section{TRANSFORMATION TO THE $C M T_{1}$ SYSTEM}

Once the complete set of combined measurements had been spliced together onto a homogeneous internal photometric system, the last task was to transform the instrumental magnitudes $(c, m, r)$ onto the standard $C M T_{1}$ system. (From this point on we drop any references to the filters and now use capital letters to refer to the magnitudes on the standard Washington system.) As already discussed, none of our raw observations were taken under photometric conditions, and later attempts to obtain supplementary imaging of fundamental Washington system standards were not successful either.

To establish the absolute photometric transformation, we have therefore returned to the data of H92. The imaging observations described by $\mathrm{H} 92$ were taken on two photometric

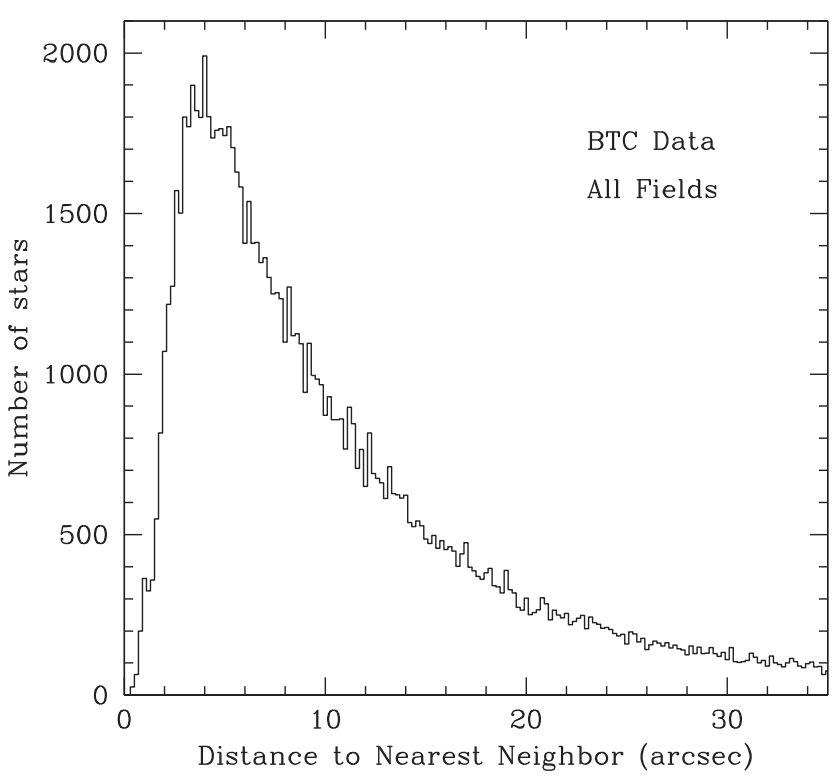

FIG. 6.-Histogram of nearest-neighbor distances from all objects in the measured database. No selection by magnitude or field location has been made. Almost all objects are uncrowded, with nearest neighbors more than $2^{\prime \prime}$ away.

nights, and the conversions of their CCD aperture photometry to the standard system are well determined (see their Table 2 for the conversion relations). To set up a network of secondary standard stars across our combined BTC fields, we selected six of the H92 fields located far enough away from the galaxy center to minimize any concerns over crowding and background light from the bulge region. These fields are the ones labelled $\mathrm{C} 1,27,34,37,43$, and 47 in their paper (see Figs. 1 and 2 of $\mathrm{H} 92$ for finder charts and field locations).

Starting from the original (i.e., H92) $C M T_{1}$ CCD images of these six small fields, we ran the normal DAOPHOT FIND/ PHOT/ALLSTAR measurement sequence to isolate and measure two to three dozen moderately bright stars on each one. A single iteration of "cleaning" (removal of faint neighbors by DAOPHOT/SUBSTAR) was performed, then a final round of aperture photometry through the $5^{\prime \prime}$ fiducial aperture employed by $\mathrm{H} 92$. Their true $C M T_{1}$ magnitudes were then calculated from the transformation equations given by $\mathrm{H} 92$. Finally, these stars were cross-identified by coordinate matching to our BTC database, and their instrumental magnitudes ( $\mathrm{cmr}$ ) were plotted against both $C-T_{1}$ and $M-T_{1}$. The results, for a total of 169 stars on six fields combined, are shown in Figures 7 and 8.

Linear least-squares fits for all three correlations against both color indices were derived and are shown in the figures. To determine the least-squares fits we rejected stars lying more than $\pm 0.1 \mathrm{mag}$ from the fiducial lines: on closer inspection, many of these turned out to be slightly nonstellar (in some cases these are actually globular clusters, in others they are simply small, faint background galaxies). For these, the largeaperture photometry from the H92 images should be systematically brighter than the PSF-fitted instrumental magnitudes of the BTC photometry, causing them to scatter upward to positive $\Delta m$ values in the graphs. For some other stars, the source of the discrepancy is unclear, except to note that most of the stars affected are the fainter ones in the sample and thus less secure. 

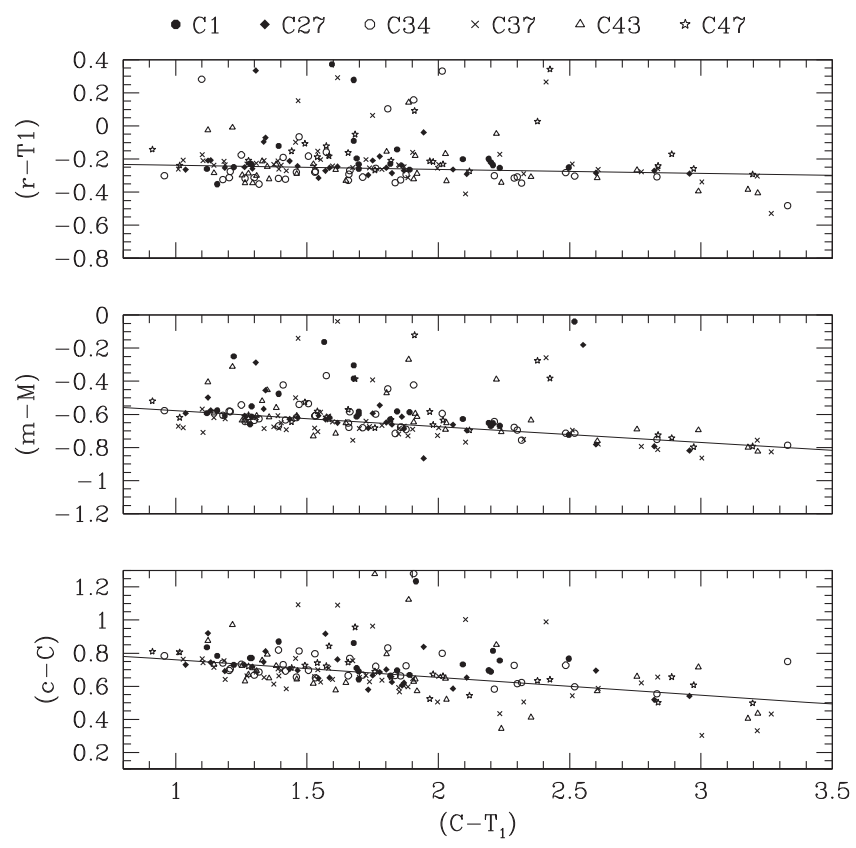

FIG. 7.-Calibration of the BTC photometry against $C-T_{1}$. Here $\left(C, M, T_{1}\right)$ are the Washington-system magnitudes for stars in six of the NGC 5128 halo fields as measured in Harris et al. (1992) and described in the text, while $(c, m, r)$ are the raw instrumental magnitudes in our BTC data. The differences $r-T_{1}$, etc., are plotted against $C-T_{1}$ color index. Each of the six fields is labelled with a different symbol type as noted at the top of the figure. The best-fitting linear least-squares relations, whose equations are given in the text, are drawn in.

The best-fitting linear relations plotted against $C-T_{1}$ are

$$
\begin{aligned}
r= & T_{1}-(0.214 \pm 0.011)-(0.024 \pm 0.006) \\
& \times\left(C-T_{1}\right)(\sigma=0.060), \\
m= & M-(0.482 \pm 0.013)-(0.096 \pm 0.007) \\
& \times\left(C-T_{1}\right)(\sigma=0.050), \\
c= & C+(0.867 \pm 0.021)-(0.107 \pm 0.011) \\
& \times\left(C-T_{1}\right)(\sigma=0.083),
\end{aligned}
$$

while in terms of $M-T_{1}$ the best-fit solutions are

$$
\begin{aligned}
r= & T_{1}-(0.181 \pm 0.017)-(0.098 \pm 0.020) \\
& \times\left(M-T_{1}\right)(\sigma=0.056), \\
m= & M-(0.453 \pm 0.014)-(0.249 \pm 0.016) \\
& \times\left(M-T_{1}\right)(\sigma=0.083), \\
c= & C+(0.844 \pm 0.027)-(0.214 \pm 0.033) \\
& \times\left(M-T_{1}\right)(\sigma=0.091) .
\end{aligned}
$$

In practice, we used equations (4) and (5) for calibrating $r$ and $m$, which had deeper photometric limits than $c$ on most of the pointings. For the $c$ conversion we used equation (3). To the accuracy permitted by the data, we find that linear calibration relations apply well over the color ranges $0.9<C-T_{1}<3.0$ and $0.5<M-T_{1}<1.4$, which include the color range of interest for GCs. Our BTC data therefore appear to be systematically on the standard Washington system established by the $\mathrm{H} 92$ photometry to within \pm 0.02 mag external accuracy anywhere over these color intervals. The standard deviations around each best-fit line (given in parentheses after each equation), for the bright-to-intermediate magnitude range that
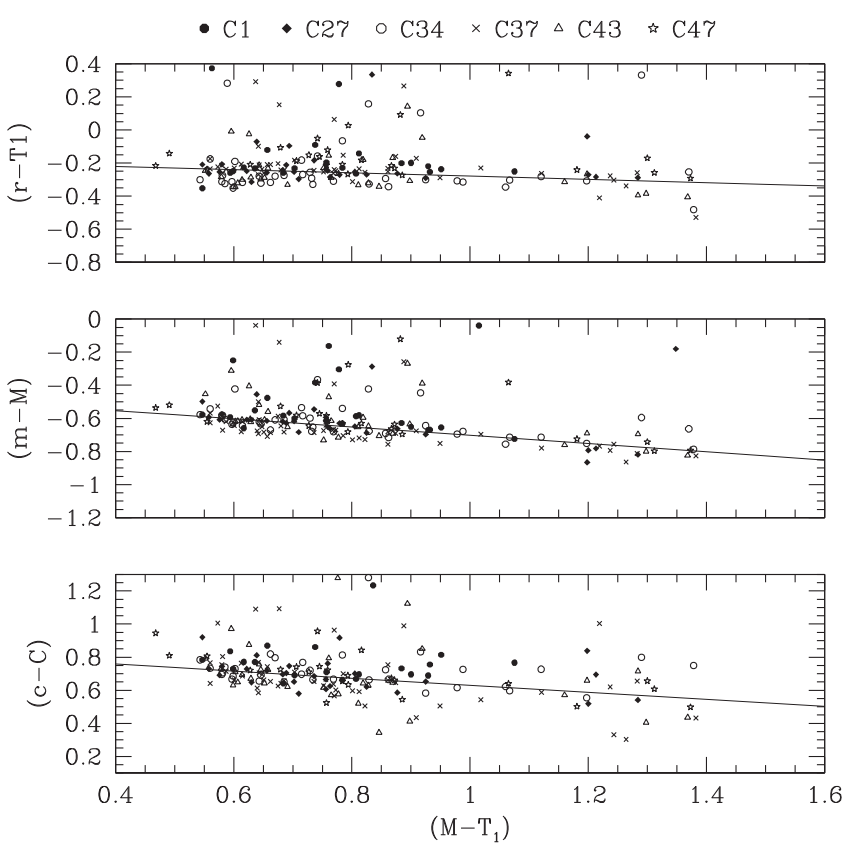

FIG. 8.-Calibration of the BTC photometry against $M-T_{1}$, with symbols as defined in the previous figure.

will be our main interest for the globular clusters, are at the level of $\sigma \simeq 0.05-0.09 \mathrm{mag}$. Since the two data sets contribute roughly equally to the observed scatter, this comparison suggests that the precision of our final BTC data set is near $\sigma \sim 0.05$ mag per star in all three filters. Encouragingly, at the level of $0.02 \mathrm{mag}$ or less, we found no indication of any systematic differences in the zero points or slopes of the transformation equations from one field to another, i.e., over a region about $30^{\prime}$ across on the BTC fields.

\section{DATA SELECTION AND COLOR-MAGNITUDE RESULTS}

The internal measurement uncertainties $e\left(C, M, T_{1}\right)$ as produced by DAOPHOT/ALLSTAR are displayed in Figure 9. We note that the plotted values are the uncertainties of each final measurement after averaging all overlapping fields, and not the uncertainty of a single measurement; because the numbers of individual measurements that were averaged together differ from one star to the next, and because the individual frames do not have the same limiting magnitude, the final $e$-values as shown in the graph show quite a bit of scatter even at the same magnitude level.

The final data set contains 81,104 objects in the "central" field box constructed from the overlapping fields and 25,936 objects in the eight outlier chips from fields $6,7,8$, and 9 . A sample of the final data is tabulated in Table 3; the full data set is available electronically from the first author on request.

The color-magnitude diagrams (CMDs) of $T_{1}$ versus $M-T_{1}$ and $C-T_{1}$ for the entire database are shown in Figures 10 and 11. Inspection of the CMDs for the individual pointings indicated no systematic offsets in magnitude or color between the various fields. The CMD, as suggested above, is completely dominated by foreground field stars, as well as faint slightly nonstellar objects of all kinds, mostly background galaxies. The globular cluster population is expected to appear in the broad color range $0.4 \lesssim M-T_{1} \lesssim 1.0$ (see H92), but this same intermediate-color range is also heavily populated by many 

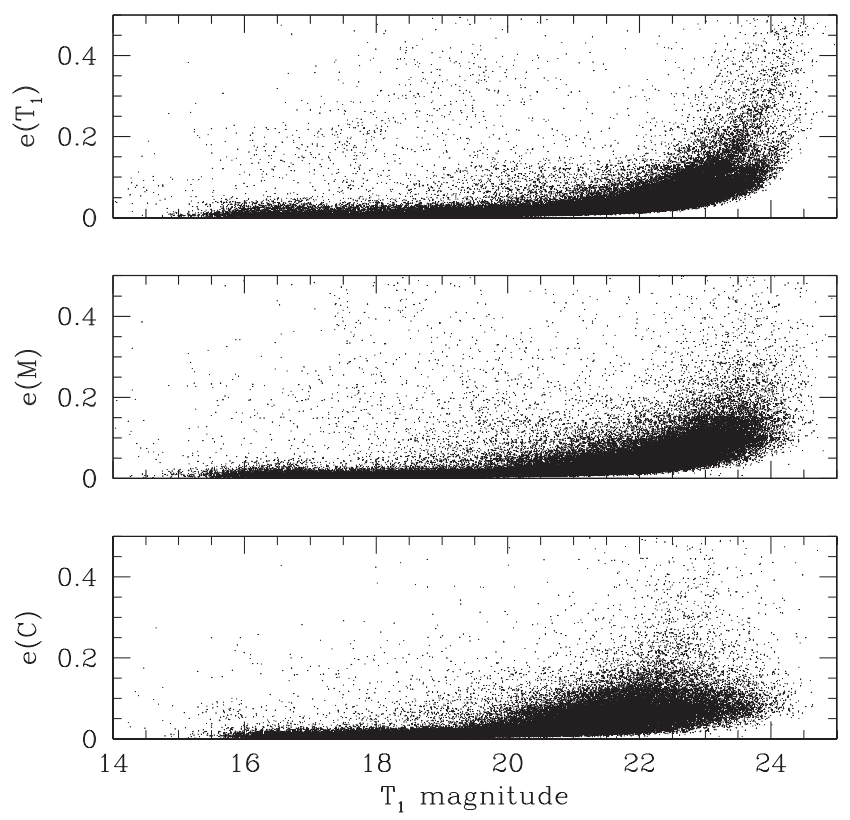

FIG. 9.- Internal DAOPHOT measurement uncertainties $e\left(C, M, T_{1}\right)$ plotted against $T_{1}$ magnitude, for 81,104 stars in the "central" region.

types of normal field stars, as well as some faint background galaxies. The many stars and faint galaxies that are redder $\left(M-T_{1}>1.0\right)$ can, however, be excluded immediately, reducing the net field contamination by a factor of 2 . We will continue this analysis in our next paper.

The standard Washington two-color diagram of $(C-M)_{0}$ versus $\left(M-T_{1}\right)_{0}$ is shown in Figure 12 for the stars brighter than $T_{1}=21$. To plot the dereddened color indices, we have used the standard relations (Geisler et al. 1991) $E(C-M)=$ $1.066 E(B-V)$ and $E\left(M-T_{1}\right)=0.900 E(B-V)$ along with our adopted foreground reddening $E(B-V)=0.11$.

\section{COMPARISON WITH PREVIOUS CLUSTER DATA}

\subsection{Washington Photometry Indices}

The calibration equations given above are derived from secondary standard stars scattered around the halo field. As an additional internal check on the magnitude scale for the NGC 5128 clusters, we have also compared our BTC magnitudes with the $C M T_{1}$ indices of 62 clusters as published in H92. The clusters themselves were discovered, by a combination of visual inspection and radial velocity measurement, in the earlier studies of Hesser et al. (1984) and Sharples (1988). We used a simple coordinate-matching procedure to find the

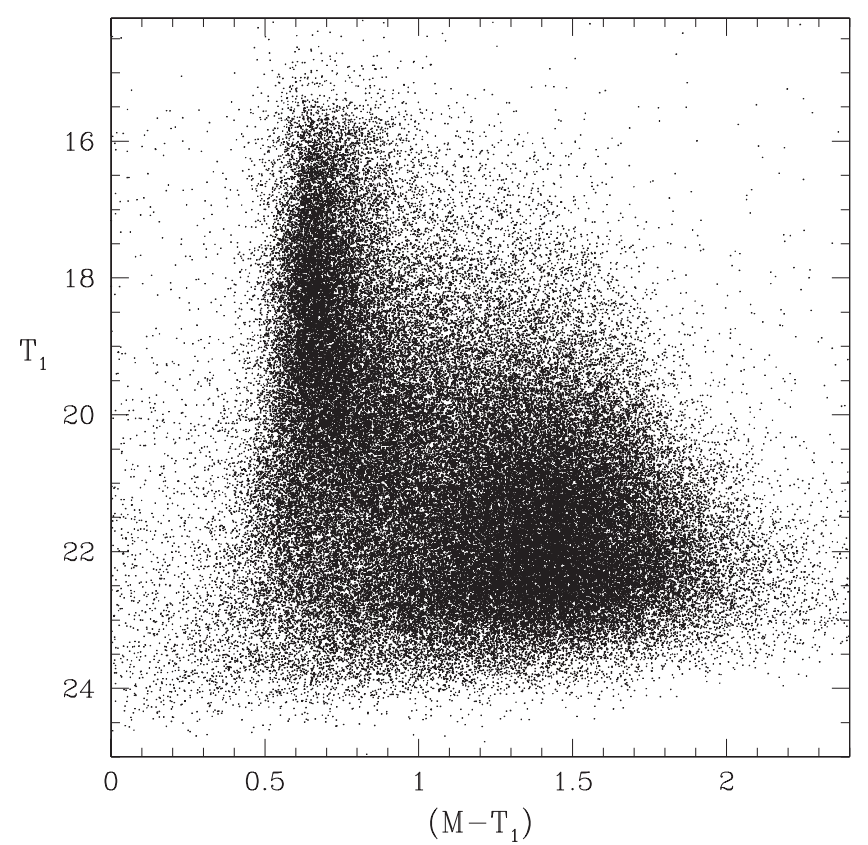

FIG. 10.-Color-magnitude diagram in $T_{1}$ vs. $M-T_{1}$ for 107,040 measured objects in the entire database. Though it contains almost all of the halo globular clusters belonging to NGC 5128 , this CMD is completely dominated by field contamination.

clusters listed in $\mathrm{H} 92$ in our BTC database; in a few cases where the BTC coordinates differed by several arcseconds from those listed in the earlier papers, we checked the identifications by visual inspection of the CCD images.

The H92 data are aperture magnitudes measured through a $5^{\prime \prime}$ radius, wide enough to contain any of the cluster profiles (see their discussion for curve-of-growth analysis and the selection of aperture size). The degree to which a cluster image matches the stellar PSF profile differs quite a bit from one object to the next, creating an extra source of internal scatter in the measured magnitudes that is not present for the calibrating stars of the same brightness. At least for the most extended (nonstarlike) cluster profiles, therefore, the H92 magnitudes should be systematically more nearly correct than the BTC data. The actual differences between the two studies are summarized in Table 4 and plotted in Figures 13 and 14. The observed scatter in all three magnitudes and three color indices is typically $\sigma \sim \pm 0.07 \mathrm{mag}$ rms, but, as noted above, occasional larger deviations are found (in Table 4 the $n$-values give the numbers of clusters used to calculate the average and standard deviation after removal of extreme outliers, i.e., greater than $3 \sigma$ from the mean magnitude difference). For

TABLE 3

BTC Data for All Measured Objects

\begin{tabular}{|c|c|c|c|c|c|c|c|c|c|}
\hline $200.19081 \ldots \ldots \ldots \ldots . . .$. & -43.15252 & 52.13 & 261.17 & 21.645 & 0.109 & 22.562 & 0.093 & 23.992 & 0.100 \\
\hline $200.19131 \ldots \ldots \ldots \ldots . . . . .$. & -43.14712 & 52.06 & 261.52 & 21.336 & 0.039 & 22.204 & 0.031 & 23.048 & 0.025 \\
\hline $200.19131 \ldots \ldots \ldots \ldots . . . . . .$. & -43.23856 & 53.14 & 255.66 & 22.669 & 0.373 & 20.569 & 0.252 & 24.209 & 0.373 \\
\hline $200.19138 \ldots \ldots \ldots \ldots . . . . . .$. & -43.21345 & 52.79 & 257.24 & 16.553 & 0.002 & 17.132 & 0.005 & 17.943 & 0.010 \\
\hline
\end{tabular}

Note.-Table 3 is presented in its entirety in the electronic edition of the Astronomical Journal. A portion is shown here for guidance regarding its form and content. 


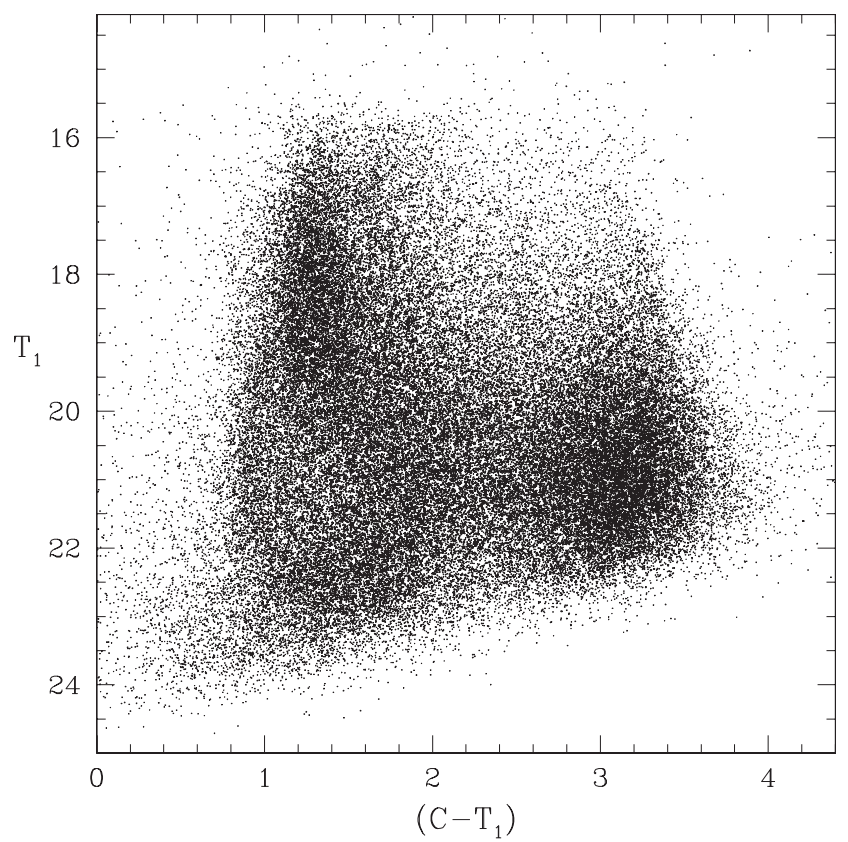

FIG. 11.-Color-magnitude diagram in $T_{1}$ vs. $C-T_{1}$ for the entire database.

$M, C, C-M$, and $M-T_{1}$ our BTC data appear to be systematically in satisfactory agreement with H92. In $T_{1}$ our (PSFfitted) BTC magnitudes tend to be slightly too faint, also making the $C-T_{1}$ color index too "blue" by $\sim 0.08$ mag. As noted above, the H92 clusters were selected in most cases by their slightly nonstellar appearance, which would be expected to make the PSF-fitted magnitudes systematically too faint, resulting in an offset in the direction shown. However, the effect shows up clearly in only the $R$ filter, and we are unable to isolate a definitive reason for this. We believe that it is likely to be

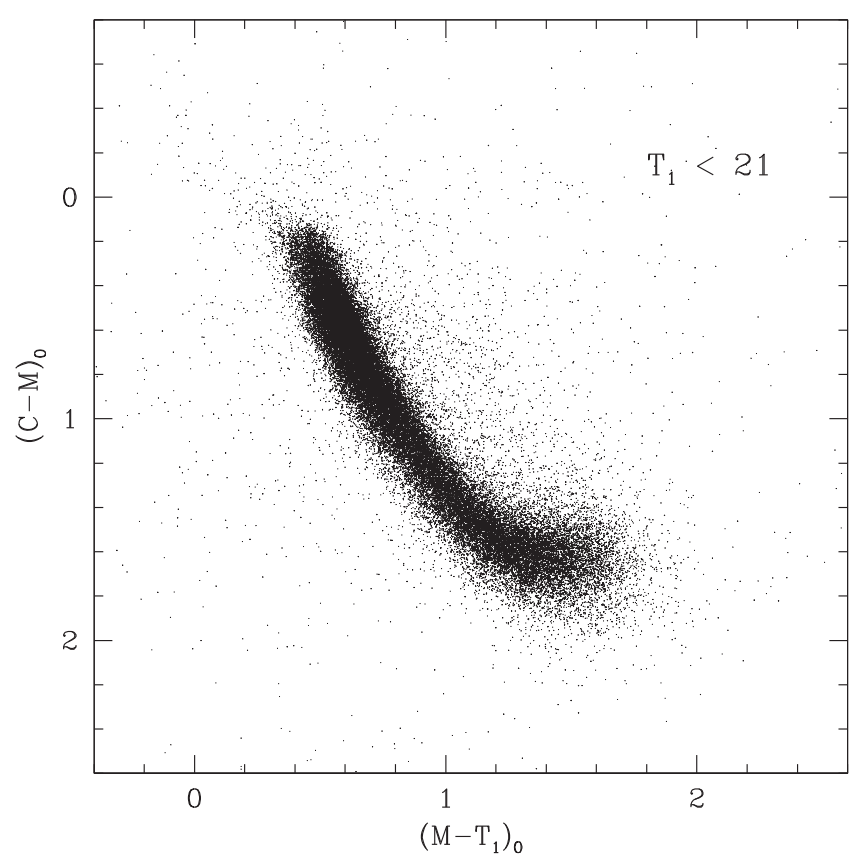

FIG. 12.-Color-color relation for the dereddened indices, $(C-M$, $M-T_{1}$ ), for a total of 51,000 stars brighter than $T_{1}=21.0$.
TABLE 4

Comparison of Cluster Photometry with Previous $C M T_{1}$ РноTOMETRY

\begin{tabular}{|c|c|c|c|}
\hline Index & $n$ & $\begin{array}{c}\text { Mean } \\
\text { (BTC-H92) }\end{array}$ & $\mathrm{rms}$ \\
\hline 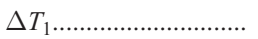 & 51 & -0.077 & 0.086 \\
\hline$\Delta M$ & 52 & -0.038 & 0.074 \\
\hline$\Delta C \ldots \ldots \ldots \ldots \ldots \ldots \ldots \ldots \ldots \ldots$ & 51 & -0.003 & 0.083 \\
\hline$\Delta(C-M) \ldots \ldots \ldots \ldots \ldots \ldots$ & 51 & +0.041 & 0.048 \\
\hline$\Delta\left(M-T_{1}\right) \ldots \ldots \ldots \ldots \ldots \ldots$ & 49 & +0.037 & 0.069 \\
\hline$\Delta\left(C-T_{1}\right) \ldots \ldots \ldots \ldots \ldots \ldots$ & 49 & +0.080 & 0.089 \\
\hline
\end{tabular}

due to a combination of the image quality limitations mentioned above, affecting the brightest objects in $R$, coupled with the nonstellar profiles and the fact that the $R$ images contain the most flux of the three filters. In any case, any photometric bias on the vast majority of fainter clusters is expected to be smaller. In our subsequent analysis of the data, we will use the BTC magnitudes and colors without applying these small offsets.

We note again that various external factors (seeing variations, weather, camera scale distortions, multiple splicing steps) put limitations on the precision and homogeneity of the database that we have not been able to completely circumvent. The combined data do, however, provide a sensible basis for statistical characteristics of the globular cluster system, as well as selection of cluster candidates for later spectroscopic observations.

\subsection{Coordinate Comparisons}

As a final test of the accuracy of our object coordinates, we compare our data with those of four previous studies of the
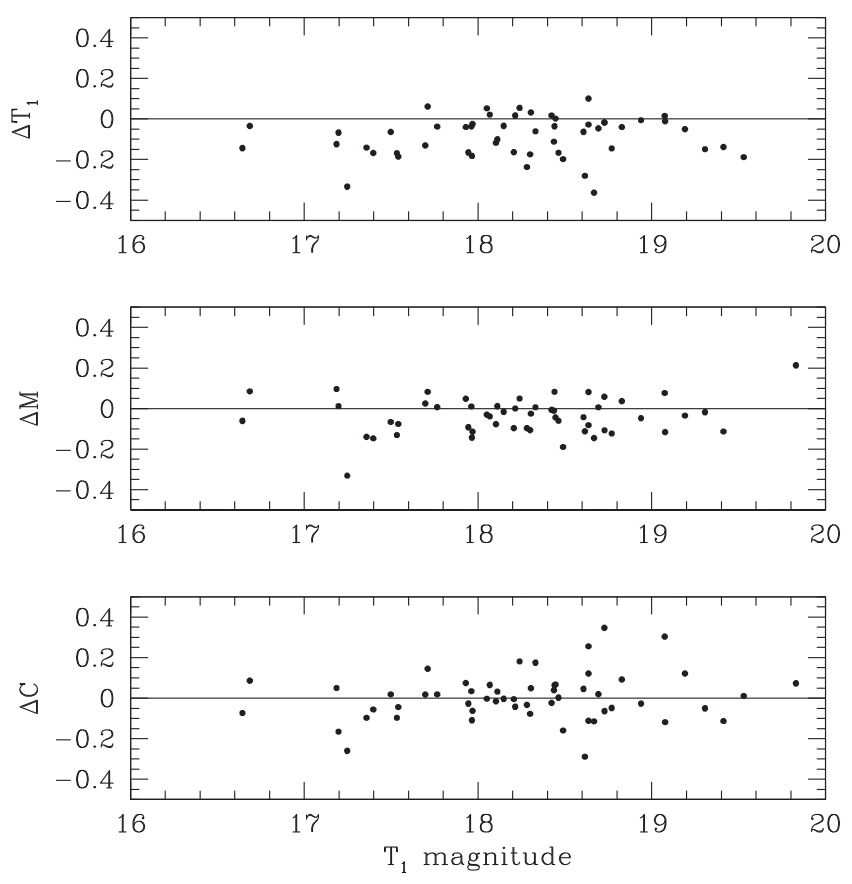

FIG. 13.-Comparison of our BTC-measured magnitudes with those of $\mathrm{H} 92$ for objects known to be globular clusters in the NGC 5128 halo. The differences $\Delta T_{1}, \Delta M, \Delta C$ are in the sense (BTC minus H92), and they are plotted against $T_{1}$ (BTC). 

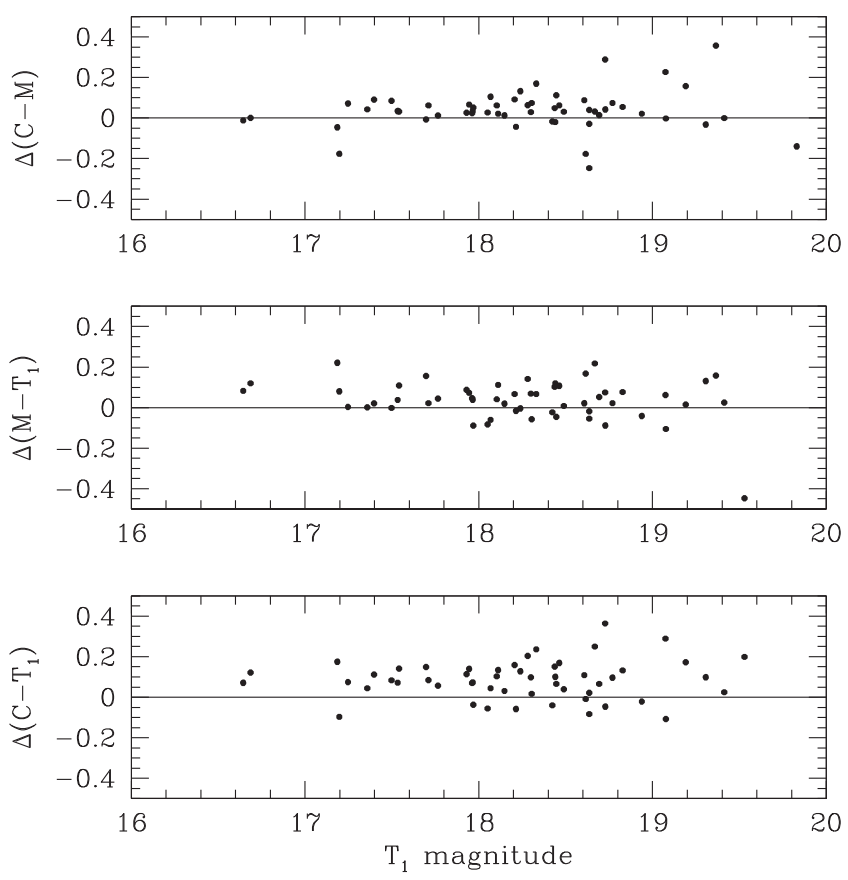

FIG. 14.-Comparison of our BTC-measured color indices with those of H92 for known globular clusters.

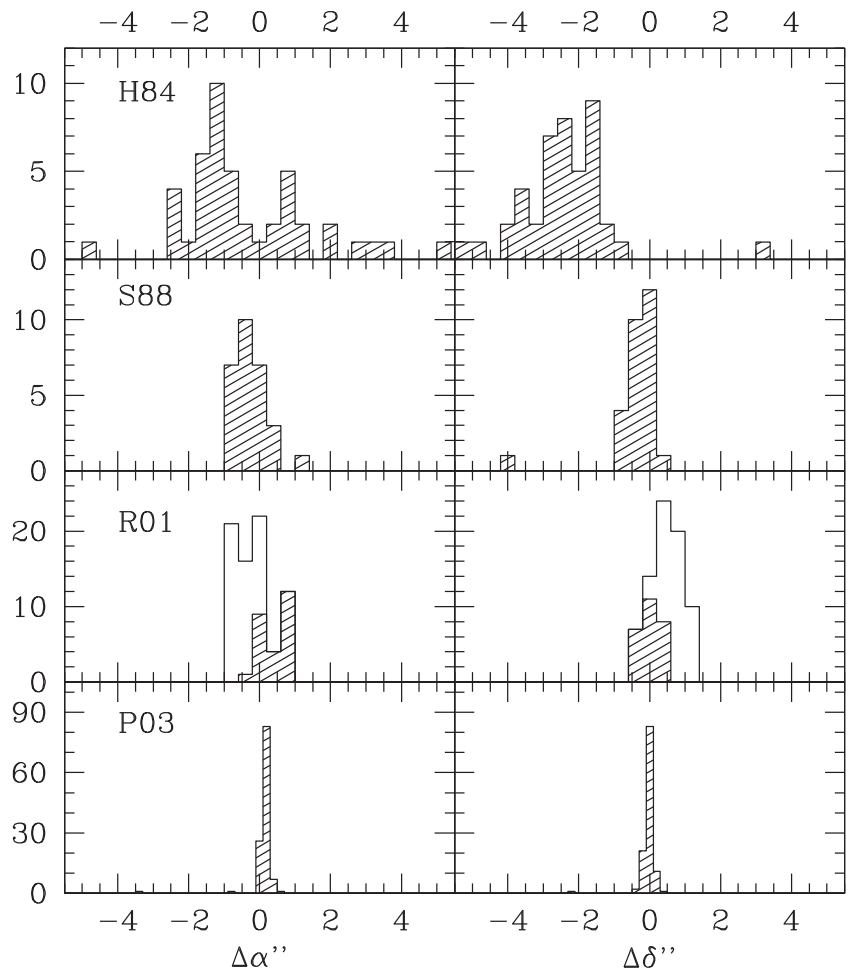

FIG. 15.- Histograms of coordinate residuals for known globular clusters, where $\Delta \alpha, \Delta \delta=$ (previous data minus BTC data) in seconds of arc. From top to bottom, the sources of previous coordinates are labelled H84 (Hesser et al. 1984), S88 (Sharples 1988), R01 (Rejkuba 2001), and P02 (Peng 2003). In the third panel, R01 field 1 is the shaded histogram and field 2 is unshaded.
TABLE 5

Comparison of Cluster Coordinate Measurements with Previous Studies

\begin{tabular}{|c|c|c|c|c|c|}
\hline Data Source & $n$ & $\begin{array}{c}\langle\Delta \alpha\rangle \\
(\operatorname{arcsec})\end{array}$ & $\sigma_{\alpha}^{\prime \prime}$ & $\begin{array}{c}\langle\Delta \delta\rangle \\
(\operatorname{arcsec})\end{array}$ & $\sigma_{\delta}^{\prime \prime}$ \\
\hline Hesser et al. (1984) .............. & 45 & -0.48 & 1.40 & -2.45 & 1.05 \\
\hline 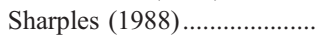 & 28 & -0.34 & 0.38 & -0.25 & 0.34 \\
\hline Rejkuba (2001) f1 .................. & 26 & +0.46 & 0.41 & +0.05 & 0.30 \\
\hline Rejkuba (2001) f2 ................... & 49 & -0.44 & 0.30 & +0.69 & 0.31 \\
\hline 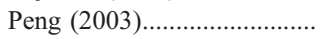 & 118 & +0.17 & 0.11 & -0.02 & 0.11 \\
\hline
\end{tabular}

NGC 5128 globular clusters: Hesser et al. (1984), Sharples (1988), Rejkuba (2001), and Peng (2003). Rejkuba (2001, hereafter R01) has used VLT ground-based imaging in two small halo fields of NGC 5128 to isolate a sample of 78 candidate globular clusters on the basis of their image morphology, along with photometry in $U$ and $V$. We have cross-identified objects in the Rejkuba lists with those in our master BTC list and find 74 of them to within a $6^{\prime \prime}$ matching radius. Peng (2003) has identified a list of 138 clusters from radial velocity measurement (36 of which overlap with previous studies). Of these, we have cross-identified 118 in our BTC list to within a $5^{\prime \prime}$ matching radius.

In Figure 15 we plot the coordinate differences between our BTC astrometry and these four previous sources. The mean and standard deviation of the residuals in each case are listed in Table 5, dramatically showing the clear improvement in coordinate measurement over the years. The positions from the studies of the early 1980s shown in the top panel (Hesser et al. 1984) were based on measurements made from widefield photographic plates with far fewer reference stars than used in the contemporary WCS solutions, and typical deviations of $2^{\prime \prime}-4^{\prime \prime}$ are seen to occur relative to the modern solutions. The declination scale of these older data is clearly displaced by $2^{\prime \prime}-3^{\prime \prime}$ relative to any of the modern data. For Sharples (1988) and Rejkuba (2001), offsets of \pm 0.5 or less are representative with only a few larger deviations, though the coordinate systems of R01 fields 1 and 2 appear to be displaced relative to one another by about an arcsecond. Finally, for the Peng (2003) data the residuals relative to our BTC grid are almost all less than a quarter of an arcsecond (corresponding to about half a BTC pixel). It is clear from the histograms that the BTC and Peng data are within $\sim 00^{\prime \prime} 1$ of being on the same absolute coordinate system, with internal scatter also at the \pm 0 "' 1 level.

The mean systematic differences $(\langle\Delta \alpha\rangle,\langle\Delta \delta\rangle$ in arcseconds) and the standard deviations of the scatter about these means are listed in Table 5. These comparisons suggest, in line with our WCS solutions described above, that the external accuracy of the BTC coordinates appears to be no worse than \pm 0 ". 2 along either axis and approaches 0 ". 1 accuracy. We recommend using the present coordinates in preference to any lists published pre-2002.

\section{SUMMARY}

This paper presents the first wide-field, multicolor photometric database suitable for global study of the NGC 5128 globular cluster system. Our data consist of Washington $C M T_{1}$ photometric indices for approximately 106,000 objects within a field of greater than 1.2 diameter centered on NGC 5128 , and with partial coverage extending to $1^{\circ} .7$. From both internal and external tests, our coordinates for the measured 
objects are shown to be accurate to well within \pm 0 "' 2 . The limiting magnitude of the data differs slightly from one location to another, but it appears to be highly complete to $T_{1} \simeq 22.0$ (equivalent to $V \simeq 22.5$ ), more than a magnitude fainter than the expected level of the classic GCLF "turnover" point.

In Paper II we discuss other aspects of the GCS of this unique galaxy, including the global spatial distribution, the total cluster population, the luminosity distribution (GCLF), and the metallicity distribution function (MDF).
We thank Scott Brooks for his technical assistance. This work was supported by the Natural Sciences and Engineering Research Council of Canada through research grants to G. L. H. H. and W. E. H., and summer fellowships to M. R. and M. M. We are pleased to acknowledge the hospitality and support at Mount Stromlo Observatory (Research School of Astronomy and Astrophysics, Australian National University) during research leaves when this paper was drafted. D. G. gratefully acknowledges support from the Chilean Centro de Astrofísica FONDAP No. 15010003.
Davidge, T. J., \& van den Bergh, S. 2001, ApJ, 553, L133

Geisler, D. 1986, PASP, 98, 847 1996, AJ, 111, 480

Geisler, D., Claria, J. J., \& Minniti, D. 1991, AJ, 102, 1836

Graham, J. A., \& Phillips, J. P. 1980, ApJ, 239, L97

Groth, E. G. 1986, AJ, 91, 1244

Harris, G. L. H., Geisler, D., Harris, H. C., \& Hesser, J. E. 1992, AJ, 104 613 (H92)

Harris, G. L. H., \& Harris, W. E. 2000, AJ, 120, 2423

Harris, G. L. H., Harris, W. E., \& Geisler, D. 2003, AJ, 128, 723 (Paper II)

Harris, G. L. H., Harris, W. E., \& Poole, G. B. 1999, AJ, 117, 855

Harris, G. L. H., Hesser, J. E., Harris, H. C., \& Curry, P. J. 1984a, ApJ, 287, 175

Harris, G. L. H., Poole, G. B., \& Harris, W. E. 1998, AJ, 116, 2866

Harris, H. C. Harris, G. L. H., \& Hesser, J. E. 1988, in IAU Symp. 126,

Globular Cluster Systems in Galaxies, ed. J. E. Grindlay \& A. G. D. Philip (Dordrecht: Kluwer), 205

Harris, H. C., Harris, G. L. H., Hesser, J. E., \& MacGillivray, H. T. 1984b, ApJ, 287, 185

Harris, W. E. 1983, PASP, 95, 406

2001, in Star Clusters, Saas-Fee Advanced Course 28, ed. L. Labhardt

\& B. Binggeli (New York: Springer)

Harris, W. E., \& Harris, G. L. H. 2002, AJ, 123, 3108

\section{REFERENCES}

Harris, W. E., Harris, G. L. H., Holland, S. T., \& McLaughlin, D. E. 2002, AJ, 124,1435

Hesser, J. E., Harris, H. C., \& Harris, G. L. H. 1986, ApJ, 303, L51

Hesser, J. E., Harris, H. C., van den Bergh, S., \& Harris, G. L. H. 1984, ApJ, 276, 491

Holland, S., Côté, P., \& Hesser, J. E. 1999, A\&A, 348, 418

Marleau, F. R., Graham, J. R., Liu, M. C., \& Charlot, S. 2000, AJ, 120, 1779

Peng, E. 2003, Ph.D. thesis, Johns Hopkins Univ.

Peng, E., Ford, H. C., \& Freeman, K. C. 2004, ApJ, 602, 705

Rejkuba, M. 2001, A\&A, 369, 812 (R01)

Rejkuba, M., et al. 2004, in preparation

Sharples, R. 1988, in IAU Symp. 126, Globular Cluster Systems in Galaxies, ed. J. E. Grindlay \& A. G. D. Philip (Dordrecht: Kluwer), 545

Schmidt, B. P., et al. 1998, ApJ, 507, 46

Soria, R., et al. 1996, ApJ, 465, 79

Stetson, P. B. 1992, in ASP Conf. Ser. 8, Astronomical Data Analysis Software and Systems I, ed. G. H. Jacoby (San Francisco: ASP), 289

van den Bergh, S., Hesser, J. E., \& Harris, G. L. H. 1981, AJ, 86, 24

Wittman, D. M., Tyson, J. A., Bernstein, G. M., Lee, R. W., dell'Antonio, I. P., Fischer, P., Smith, D. R., \& Blouke, M. M. 1998, Proc. SPIE, 3355, 626

Zacharias, N., et al. 2000, AJ, 120, 2131

Zepf, S. E., \& Ashman, K. M. 1993, MNRAS, 264, 611 